\title{
Lightweight Design is Undergoing Change
}

We are experiencing exciting times for lightweight design - or perhaps even the beginning of a whole new era? The Federal Government quite rightly regards lightweight design as a key technology for ensuring Germany's industrial future. The aviation sector has been demonstrating for many years how the moving mass of high-tech structures can be reduced without sacrificing safety or cost-efficiency. Owing to emissions legislation and the approaching age of electromobility, a further, powerful driver of lightweight design technology has made its presence known in recent years: automotive engineering. And it raises a question that sounds so simple and yet so multi-faceted: how can we establish lightweight design in large-scale production? The question is no longer limited to the task of lowering the weight of our cars. In reality, it is more concerned with the task of achieving the optimum relationship between low weight and at the same time inexpensive and environmentally friendly production - while establishing a cycle of materials that works. These questions are of great interest across a number of industries, since mechanical engineering, railways, shipbuilding and wind energy are crying out for lightweight, affordable and environmentally compatible structures.

As lightweight designers, we are rising to this significant challenge. We are aware that lightweight design now goes far beyond substituting materials at the component level. In 2017, lightweight design means exploiting the potential in an entire structure, an entire product or an entire vehicle. We are facing an era of system lightweight design - the development of new types of weight-orientated systems is replacing the optimisation of familiar products. The discussion about weight savings, resulting from $800-\mathrm{V}$ rather than $400-\mathrm{V}$ networks in future electric vehicles, even takes us beyond the question of the right material.

Under my predecessor, Ulrich Knorra, lightweight.design developed into a central platform for exchanging expert opinions between developers and users of lightweight technologies. I am pleased to be able to continue this tradition and to present to you a selection of the latest developments and most relevant topics every two months. However, one thing is certain: it is you who plays the most significant role in the success of this magazine.

You are the experts in the many different technologies related to lightweight design. You conduct research into new methods and develop new products for the market. I see lightweight.design as your forum, whose task it is to promote the exchange of new ideas about technical and scientific topics and products. For this reason, I cordially invite you to play a part in lightweight.design. I will be delighted to hear your suggestions for topics for specialist articles at any time.

Here's to our successful collaboration! 4 